\title{
Safety and feasibility of near-infrared image-guided lymphatic mapping of regional lymph nodes in esophageal cancer
}

Krista J. Hachey, MD, ${ }^{a}$ Denis M. Gilmore, MD, ${ }^{a}$ Katherine W. Armstrong, MPH, ${ }^{a}$ Sean E. Harris, MA, ${ }^{a}$ Jason L. Hornick, MD, PhD, ${ }^{\mathrm{b}}$ Yolonda L. Colson, $\mathrm{MD}, \mathrm{PhD},{ }^{\mathrm{a}}$ and Jon O. Wee, $\mathrm{MD}^{\mathrm{a}}$

\section{ABSTRACT}

Objective: To assess safety and feasibility of an intraoperative, minimally invasive near-infrared (NIR) image-guided approach to lymphatic mapping in patients with esophageal cancer.

Methods: Although local lymph nodes (LNs) are removed with the esophageal specimen, no techniques are available to identify the regional LNs (separate from the esophagus) during esophagectomy. We hypothesize that NIR imaging can identify regional LNs with the potential to improve staging and guide the extent of lymphadenectomy. Nine of the 10 patients enrolled had resectable esophageal adenocarcinoma and underwent NIR mapping following peritumoral submucosal injection of indocyanine green (ICG) alone or premixed in human serum albumin (ICG:HSA) before resection. NIR imaging was performed in situ and ex vivo.

Results: In 6 of the 10 patients, intraoperative NIR imaging demonstrated an NIR signal at all tumors and in 2 to $6 \mathrm{NIR}^{+}$regional LNs. $\mathrm{NIR}^{+} \mathrm{LNs}$ were not identified in 4 patients: 1 patient with occult stage IV disease, for whom further imaging was not performed and thus was excluded from analysis, and 3 patients in whom ICG was used without HSA. Identification of local LNs on the esophagus was obscured by a peritumoral background. Importantly, the pathological status of $\mathrm{NIR}^{+}$regional LNs reflected overall regional nodal status.

Conclusions: NIR lymphatic mapping is safe and feasible in patients with esophageal cancer and can identify regional LNs when ICG:HSA is used. Although more work is needed to improve background signals and local LN identification, intraoperative detection of regional $\mathrm{NIR}^{+}$LNs allows an in-depth histological analysis of LN basins not commonly scrutinized as part of the spec-

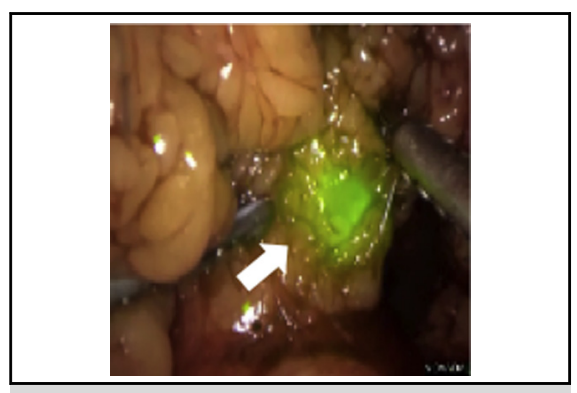

NIR-guided in vivo regional esophageal LN detection.

\section{Central Message}

Near-infrared lymphatic mapping is safe and feasible for in situ identification of regional lymph nodes in esophageal cancer.

\section{Perspective}

This "first in human" trial demonstrates preliminary safety and feasibility of intraoperative minimally invasive near-infrared lymphatic mapping for identification of regional lymph nodes in esophageal cancer. NIR imaging may have potential as a staging tool in the future to better select patients for neoadjuvant therapy and endomucosal resection.

See Editorial Commentary page 555. imen and may improve the detection of occult nodal disease. (J Thorac Cardiovasc Surg 2016;152:546-54)

Esophageal cancer is an aggressive malignancy, with an overall 5-year survival rate of only $18 \% .{ }^{1}$ Even with limited N1 disease by the current TNM staging system, 5-year survival is $<40 \%$. $^{2}$ The incidence of esophageal cancer is

From the ${ }^{\mathrm{a} D i v i s i o n}$ of Thoracic Surgery and ${ }^{\mathrm{b}}$ Department of Pathology, Brigham and Women's Hospital, Boston, Mass.

Y.L.C. and J.O.W. share senior authorship.

Funding for this research study was supported by T32 National Institutes of Health training grant (5T32CA009535) and internal hospital funds.

Received for publication Jan 5, 2016; revisions received March 27, 2016; accepted for publication April 3, 2016; available ahead of print May 12, 2016.

Address for reprints: Yolonda L. Colson, MD, PhD, Division of Thoracic Surgery, Department of Surgery, Brigham and Women's Hospital, 75 Francis St, Boston, MA 02115 (E-mail: YCOLSON@partners.org).

$0022-5223 / \$ 36.00$

Copyright (c) 2016 by The American Association for Thoracic Surgery

http://dx.doi.org/10.1016/j.jtcvs.2016.04.025 increasing, with rates of adenocarcinoma in the United States up by more than $350 \%$ over the past 20 years.

The American Joint Committee on Cancer's most recent pathological nodal staging scheme for esophageal cancer stratifies prognosis based on the total number of metastatic nodes present rather than by proximity to the tumor. ${ }^{4}$ Given that a more extensive lymphadenectomy (LAD) is associated with better prognostication but that the lymph

Scanning this QR code will take you to a procedural video. 

Abbreviations and Acronyms
$\mathrm{CT}=$ computed tomography
EUS $=$ endoscopic ultrasound
$\mathrm{GE}=$ gastroesophageal
HSA $=$ human serum albumin
ICG $=$ indocyanine green
$\mathrm{LAD}=$ lymphadenectomy
LN = lymph node
NIR $=$ near-infrared
PET $=$ positron emission tomography
SLN $=$ sentinel lymph node

nodes (LNs) that drain a specific tumor and are at risk for metastatic disease are unknown, current surgical recommendations stipulate the removal of a minimum of 20 LNs for T2 (local) disease and $\geq 30 \mathrm{LNs}$ for more advanced T3/T4 disease, to attempt to include as many LNs as possible. ${ }^{5-7}$ However, even an extensive 3 -field LAD carries a 5\% risk of recurrent disease appearing in the locoregional nodes, suggesting that key nodes can be missed despite an aggressive LAD and can contain occult nodal metastases. ${ }^{8}$ Although the more common 2-field LAD has been shown to minimize operative morbidity compared with 3 -field LAD, some studies have suggested that recurrence rates may be higher than those after an extended LAD. ${ }^{9}$

Innovative techniques to optimize intraoperative LAD have the potential to improve outcomes by sampling the specific tumor-associated LNs most likely to harbor metastatic disease and by increasing the pathological scrutiny of these nodes. The goals of such an approach are to provide more accurate prognostic information, potentially reduce the likelihood of regional nodal recurrence by removing occult disease, and identify subsets of patients that can benefit from additional adjuvant therapies or, in the case of early $\mathrm{T} 1$ disease, esophagealsparing procedures.

Over the past 10 years, efforts to improve intraoperative nodal staging in esophageal cancer have focused primarily on the application of radiocolloid tracers for sentinel LN (SLN) mapping. These efforts have not produced a reproducible technique for intraoperative in situ SLN identification, however. ${ }^{10-12}$ The frequent requirement for an open procedure with back table dissection of the esophagectomy specimen, along with exposure of patients and staff to radioactive tracers, have hindered widespread clinical translation. Finally, the inability to identify the SLN due to "shine-through" from the primary tumor makes the identification and removal of "second-tier" nodes beyond SLNs of potential prognostic benefit.

Near-infrared (NIR) imaging has emerged as a safe and promising intraoperative technology for lymphatic mapping in melanoma and breast and lung cancers. ${ }^{13-16}$
However, a single study using NIR imaging in patients with esophageal cancer required preoperative computed tomography (CT) lymphography for SLN localization rather than NIR imaging alone, and used open thoracotomy for identification and resection of nodes. ${ }^{17}$ We hypothesized that NIR imaging could be developed for minimally invasive intraoperative lymphatic mapping of tumor-draining LNs, including second-tier regional nodes beyond paraesophageal nodes, for the targeted analysis of regional nodes at risk for metastases. The clinical translation of such an approach has the potential to make oncologic LAD more attainable and, more importantly, can personalize histological scrutiny to the most critical regional LNs that identify the tumor-associated lymphatic pathway.

The aims of this pilot trial were to develop intraoperative in situ NIR image-guided lymphatic mapping in surgically resectable esophageal cancer and to evaluate the safety and feasibility of this approach. This study focuses on NIR lymphatic mapping of the regional LNs because they are not removed with the esophageal specimen and may result in the identification of additional occult nodal disease.

\section{METHODS \\ Eligibility}

Ten patients diagnosed with esophageal adenocarcinoma scheduled to undergo minimally invasive Ivor Lewis esophagectomy were enrolled between June 2013 and April 2014. This trial was approved by the Dana-Farber Cancer Institute Institutional Review Board, and all patients provided informed consent. Exclusion criteria included patients who choose not to proceed with surgery, age $<18$ years, pregnancy or breastfeeding, history of iodide or seafood allergy, and discovery of occult metastatic disease at the time of surgery. All study patients underwent preoperative chest CT, positron emission tomography (PET)/CT, and endoscopic ultrasound (EUS).

\section{NIR Image-Guided Lymphatic Mapping and Surgical Resection}

Indocyanine green (ICG) is a Food and Drug Administration-approved NIR dye with a fluorescence emission wavelength ranging from 800 to $1000 \mathrm{~nm}^{13}$ ICG signal is detectable in vivo with minimal NIR autofluorescence and background signals from normal human tissue. Based on a previous successful intrathoracic NIR dose escalation trial in lung cancer, ${ }^{13}$ an ICG dose of $2.5 \mathrm{mg}$ was used for lymphatic mapping in this trial. The ICG (Novadaq Technologies, Bonita Springs, Fla) at an initial stock dose of $25 \mathrm{mg}$ was diluted to $2.5 \mathrm{mg} / \mathrm{mL}$ with either sterile water (ICG alone) or $25 \%$ human serum albumin (ICG:HSA), because albumin has been shown to increase the effective hydrodynamic diameter of ICG by almost 6-fold, resulting in increased lymphatic retention. ${ }^{18}$

Real-time NIR fluorescence images were obtained intraoperatively with the Novadaq PINPOINT minimally invasive endoscopic fluorescence imaging system using a 10-mm, 30-degree NIR thoracoscopic camera. Three simultaneous images of the surgical field were collected: a standard visible light image, a black and white NIR image, and a merged image with the NIR signal shown in green in the context of a visible light image. Detection was by qualitative color visualization, because software for signal-to-background ratio calculation and semiquantitative intensity evaluation was not available for this study.

Patients were randomized in block cohorts of 2 to receive ICG $(n=5)$ or ICG:HSA $(n=5)$ injected peritumorally via endoscopy before being 


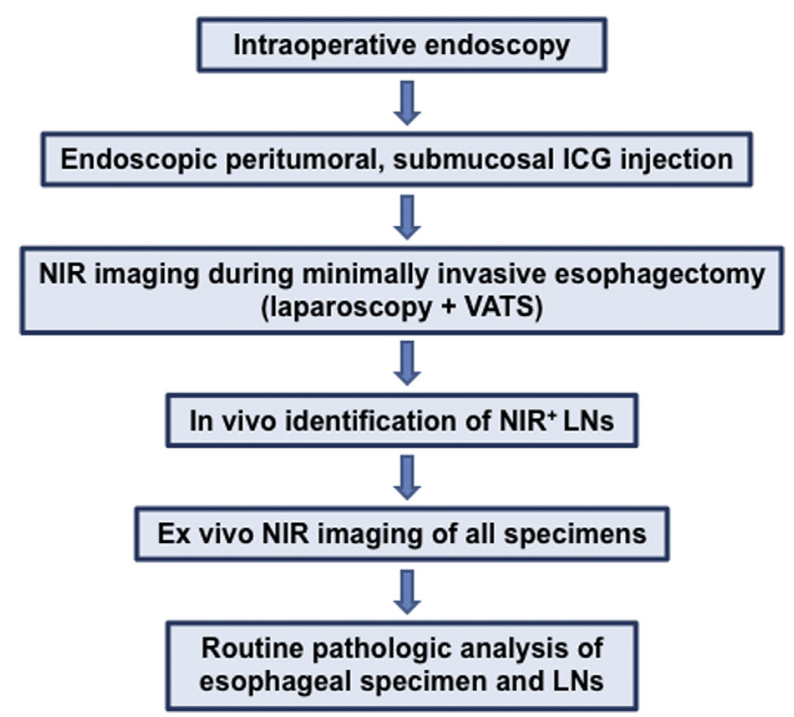

FIGURE 1. Intraoperative NIR imaging protocol for lymphatic mapping in esophageal cancer. ICG, Indocyanine green; NIR, near-infrared; VATS, video-assisted thorascopic surgery; $L N$, lymph node.

positioned for esophagectomy (Figure 1). A total of $1 \mathrm{~mL}$ of ICG or ICG:HSA was administered peritumorally via 4-corner submucosal injection adjacent to each lesion. In some cases, an endoluminal mass was not evident on intraoperative endoscopic owing to a previous endoscopic lesion resection or neoadjuvant treatment response. The previous location of the mass as determined by preoperative staging endoscopy as well as mucosal irregularities consistent with neoadjuvant treatment response guided the accuracy of dye injection in these cases. NIR imaging was performed throughout both the laparoscopic and thoracoscopic portions of each case. Patients were monitored throughout for signs of anaphylaxis or other adverse events, none of which occurred.

All patients with localized disease $(n=9)$ underwent a minimally invasive Ivor Lewis esophagectomy with 2 -field LAD. In 4 patients, robotic assistance was provided for the thoracic portion of the procedure. The NIR videoscope was used during dissection of each nodal basin to assess for $\mathrm{NIR}^{+}$nodes. All cases began with the laparoscopic abdominal portion except in the case of patient 1 , who underwent left video-assisted thoracoscopy surgery (VAT) for LN staging and NIR imaging of a PET avid focus before the abdominal dissection and subsequent esophagectomy.

\section{Pathological Analysis}

LNs removed with the esophageal specimen were dissected during gross pathological analysis and were considered "local" LNs. In contrast, LNs identified intraoperatively at discrete stations away from the esophagus (eg, pericardial, gastric, celiac stations) were defined as "regional" LNs. Location, including discrete stations, and NIR status were recorded for all LNs before they were sent for independent pathological analysis and interpretation.

Immunohistochemistry for micrometastatic disease in $\mathrm{NIR}^{+} \mathrm{LNs}$ was performed on $4-\mu \mathrm{m}$-thick formalin-fixed, paraffin-embedded $\mathrm{LN}$ tissue sections following proteinase antigen retrieval using a cocktail of mouse monoclonal anti-keratin antibodies (AE1/AE3; 1:200 dilution, 40-minute incubation; Dako, Carpinteria, Calif) and the EnVision + System-HRP (Dako).

\section{RESULTS}

\section{Patient Demographics}

A total of 10 patients ( 9 males and 1 female) were enrolled and underwent endoscopic peritumoral ICG injection. Two patients (patients 5 and 9) had T1 disease and underwent surgery without neoadjuvant therapy (Table 1). Patient 5 had undergone previous endoscopic mucosal resection of an intramucosal adenocarcinoma before enrollment, and underwent esophagectomy for persistent multifocal high-grade dysplasia. The other 8 patients were assessed to have locally invasive disease and received neoadjuvant chemoradiation, although 1 patient was found to have occult stage IV disease intraoperatively and did not undergo resection, and thus was excluded from the NIR analysis.

The mean patient age was $67 \pm 7.62$ years. Patient demographic information, including histology, clinical stage, tumor location, and subsequent pathological stage at resection, is presented in Table 1. Lesions were considered lower-third/gastroesophageal (GE) junction tumors in all patients except patient 5, who had an intramucosal lesion at $30 \mathrm{~cm}$ in the middle third of the esophagus.

Preoperative PET/CT was node-negative in 8 of 10 patients, 5 of whom had undergone neoadjuvant chemoradiotherapy for suspected N1 disease by EUS or initial PET/CT. Two patients demonstrated new or persistent PET avidity at the time of surgery. Patient 1 was initially clinical stage T2N0, underwent neoadjuvant chemoradiation, and subsequently developed avidity in the left mediastinum, correlating with an LN between the left mainstem bronchus and aorta. The initial PET/CT for patient 10 demonstrated avidity in the region of the gastrohepatic ligament with decreased but persistent avidity following neoadjuvant chemoradiation.

\section{Feasibility of Submucosal Peritumoral NIR Lesion Marking and the Importance of Tracer Composition}

In this pilot study, we evaluated the feasibility and safety of endoscopic submucosal injection of ICG dye (Figure 2), which, unlike the subadventitial approach, permits direct visualization of the lesion at the time of operative endoscopy and facilitates accurate peritumoral NIR marking. Furthermore, because lymphatic mapping requires that the tumor-associated lymphatic pathway remains intact, we sought to avoid the potential lymphatic disruption that can occur with dissection and subadventitial injection.

Sclerotherapy needles were primed with ICG before injection, to avoid inadequate dose delivery. ICG injection was performed via endoscopy under direct visualization in a 4-corner peritumoral manner in all patients without difficulty. No adverse events were noted.

To assess the importance of the ICG carrier for NIR lymphatic mapping, patients undergoing minimally invasive esophagectomy were randomized to endoscopic peritumoral injection of ICG mixed with saline (ICG alone; $\mathrm{n}=5$ ) or ICG mixed with HSA (ICG:HSA; $\mathrm{n}=5$ ). Endoscopic peritumoral ICG injection was successfully 
TABLE 1. Description of subjects by diagnosis, stage, and esophageal tumor location

\begin{tabular}{|c|c|c|c|c|c|}
\hline Patient & $\begin{array}{c}\text { Location of } \\
\text { adenocarcinoma, } \mathbf{c m} \dagger\end{array}$ & $\begin{array}{l}\text { Clinical stage } \\
\text { at diagnosis } \ddagger\end{array}$ & $\begin{array}{c}\text { Neoadjuvant } \\
\text { chemoradiation }\end{array}$ & $\begin{array}{c}\text { Posttreatment } \\
\text { cN stage } \S\end{array}$ & $\begin{array}{c}\text { Postoperative } \\
\text { pathological stage }\end{array}$ \\
\hline 1 & $42-45$ & $\mathrm{~T} 2 \mathrm{~N} 1$ & Yes & N1 & T2NO \\
\hline 2 & $31-38$ & T3N1 & Yes & No & T3NO \\
\hline 3 & 39 & $\mathrm{~T} 3 \mathrm{~N} 1$ & Yes & No & $\mathrm{T} 2 \mathrm{~N} 1$ \\
\hline 4 & $35-42$ & $\mathrm{~T} 3 \mathrm{~N} 1$ & Yes & No & T3NO \\
\hline 5 & 30 & T1N0 & No & - & T1N0 \\
\hline $6^{*}$ & $34-44$ & $\mathrm{~T} 2 \mathrm{~N} 1$ & Yes & No & Stage IV due to M1 \\
\hline 7 & 42 & TxNO & Yes & No & T3N1 \\
\hline 8 & $34-40$ & T3N1 & Yes & No & T3N1 \\
\hline 9 & 35 & T1N0 & No & - & $\mathrm{T} 1 \mathrm{~N} 1$ \\
\hline 10 & $39-44$ & TxN1 & Yes & N1 & $\mathrm{T} 3 \mathrm{~N} 3$ \\
\hline
\end{tabular}

*This patient was excluded owing to occult stage IV disease identified at the time of laparoscopy. $\nmid$ Location of esophageal mass based on preoperative endoscopic ultrasound (EUS). $\ddagger$ Clinical stage at time of diagnosis based on EUS and positron emission tomography (PET)/computed tomography (CT) findings. $§$ Post-neoadjuvant treatment clinical $\mathrm{N}$ stage is based on follow-up restaging PET/CT before operative intervention.

performed in all cases $(n=10)$, and in situ detection of NIR signal at the site of the esophageal tumor was achieved in all cases. The time from injection to initial videoscopic NIR imaging ranged from 20 minutes to 1 hour and 20 minutes. Patient 6 underwent peritumoral ICG:HSA injection of a clinically staged T2N0 tumor but was subsequently excluded from nodal analysis after occult stage IV disease was discovered during laparoscopy. Given that esophagectomy and LAD were not performed and NIR imaging was not completed, this patient was excluded from further analysis. Of the 9 patients who underwent esophagectomy and LAD, ICG:HSA was the superior lymphatic imaging agent, with identification of regional LNs in $100 \%$ (4 of 4 patients), compared with $40 \%$ ( 2 of 5 patients) using ICG alone. No adverse events were noted in any patient.

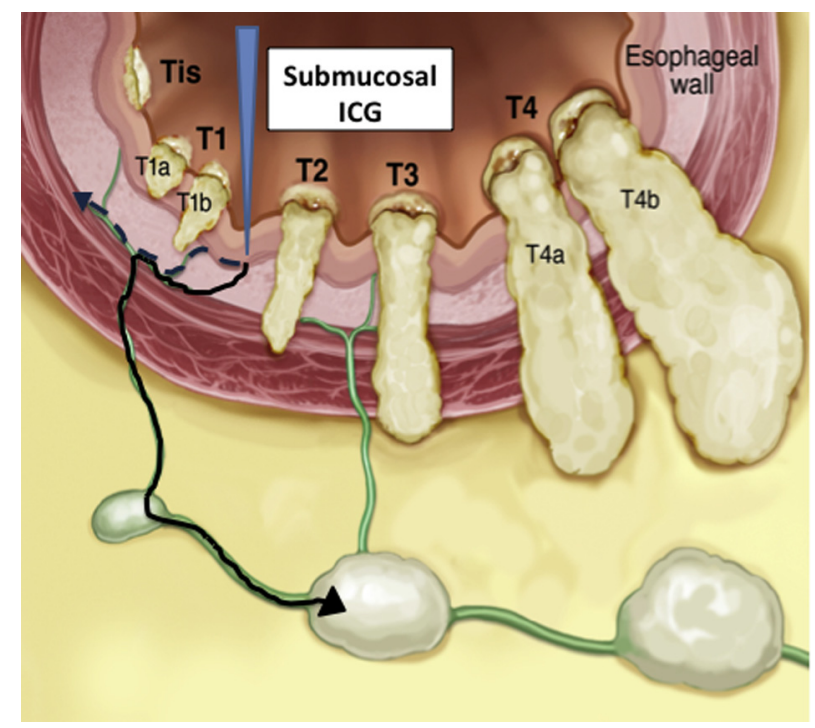

FIGURE 2. Submucosal esophageal ICG injection approach resulting in ICG dye migration through submucosal lymphatics (dark-blue line) and to more distal regional LNs (black line). ICG, Indocyanine green.

\section{Results of Intraoperative NIR Lymphatic Mapping}

The mean total number of LNs resected (including both local and regional nodes) for $\mathrm{T} 1$ and $\mathrm{T} 2 / \mathrm{T} 3$ surgical specimens was $21.5 \pm 4.95$ and $29.29 \pm 5.99$, respectively. The total number of local LNs removed with the esophageal specimen and dissected at the time of formal pathological analysis ranged from 15 to 30 per specimen (Table 2). The total number of regional LNs identified intraoperatively as separate from the esophageal specimen and removed ranged from 2 to 11 .

Significant NIR background directly on the esophageal specimen, particularly around the injection site, was observed. No distinct $\mathrm{NIR}^{+}$local LNs were identified directly on the specimen, owing to the high peritumoral ICG signal; however, between 2 and $6 \mathrm{NIR}^{+}$regional LNs, separate from the esophagus, were readily identified in 6 of the 9 patients. All 3 patients in whom regional $\mathrm{NIR}^{+}$LNs were not identified had received an injection of ICG alone, confirming the superiority of ICG:HSA, as regional $\mathrm{NIR}^{+}$nodes were identified in 4 of 4 patients undergoing esophagectomy. ICG nodal uptake was confirmed ex vivo on all identified $\mathrm{NIR}^{+}$LNs (Figure 3). Bisection of $\mathrm{NIR}^{+}$LNs demonstrated tracer uptake throughout the nodal parenchyma, indicative of lymphatic migration and not surface contamination.

The time from ICG injection to video-assisted thorascopic surgery (VATS) ranged from 39 minutes (when VATS was performed before laparoscopy) to 6 hours and 18 minutes. Intrathoracic NIR imaging showed evidence of dye migration on the esophagus and dye extravasation into the posterior mediastinum in some cases. These findings did hinder assessment of LNs directly on the esophagus; however, regional LNs within the mediastinum could still be visualized without a background NIR signal.

In vivo identification of $\mathrm{NIR}^{+} \mathrm{LNs}$ was feasible as early as 30 minutes and as late as 3.5 hours. $\mathrm{NIR}^{+}$regional LNs were identified at $\mathrm{LN}$ stations in both the abdominal and thoracic 
TABLE 2. Characteristics of lymphadenectomy specimens including $\mathrm{NIR}^{+}$regional LNs

\begin{tabular}{|c|c|c|c|c|c|c|c|}
\hline \multirow[b]{2}{*}{ Patient } & \multirow[b]{2}{*}{ NIR tracer } & \multicolumn{2}{|c|}{ Time to first imaging, $\min$} & \multicolumn{2}{|c|}{ Total LNs removed } & \multirow[b]{2}{*}{$\mathrm{NIR}^{+}$regional LNs } & \multirow[b]{2}{*}{ Nodal station of $\mathrm{NIR}^{+} \mathrm{LNs}^{*}$ (time to detection) } \\
\hline & & Lap & VATS & Local & Regional & & \\
\hline 1 & ICG:HSA & - & 39 & 30 & 10 & 6 & 8L (NA), 16 (NA) \\
\hline 2 & ICG:HSA & 60 & - & 20 & 2 & 2 & 7 (NA) \\
\hline 3 & ICG alone & 60 & 222 & 15 & 11 & 0 & - \\
\hline 4 & ICG alone & 26 & 192 & 25 & 3 & 0 & - \\
\hline 5 & ICG alone & 80 & 378 & 16 & 2 & 0 & - \\
\hline 7 & ICG alone & 34 & 275 & 28 & 5 & 1 & $16(80 \mathrm{~min})$ \\
\hline 8 & ICG:HSA & 25 & 240 & 17 & 8 & 2 & $16(30 \mathrm{~min}), 20(60 \mathrm{~min})$ \\
\hline 9 & ICG:HSA & 20 & 265 & 15 & 10 & 3 & $16(90 \mathrm{~min}), 17(110 \mathrm{~min})$ \\
\hline 10 & ICG alone & 45 & 310 & 25 & 6 & 4 & 16 (205 min), $17(210 \mathrm{~min})$ \\
\hline
\end{tabular}

Local LNs are located directly on the esophageal specimen; regional LNs are separate from the specimen. NIR, Near-infrared; VATS, video-assisted thorascopic surgery; $L N$, lymph node; $N I R^{+}$, detection of near-infrared fluorescence; ICG:HSA, indocyanine green premixed with human serum albumin; $N A$, not applicable; ICG, indocyanine green. *Regional LN stations: 7, subcarinal; 8L, thoracic lower periesophageal; 16, pericardial; 17, left gastric; 20, celiac axis (see Figure 4).

cavities, including the pericardial $(\mathrm{n}=5)$, left gastric $(\mathrm{n}=2)$, celiac axis $(\mathrm{n}=1)$, lower periesophageal $(\mathrm{n}=1)$, and subcarinal $(\mathrm{n}=1)$ stations (Figure 4). Cervical LNs were sampled in 1 patients and showed no evidence of NIR uptake.

\section{Histopathological Analysis}

Routine histopathological analysis of regional LNs distinct from the esophageal specimen demonstrated that all $\mathrm{NIR}^{+}$regional LNs were negative for disease, which was consistent with the pathological status of all other regional LNs removed (Table 3). Immunohistochemistry for keratins was also negative in regional $\mathrm{NIR}^{+} \mathrm{LNs}$, indicating the absence of micrometastatic disease in these regional $\mathrm{NIR}^{+} \mathrm{LNs}$ as well. Pathological local LNs were identified in 5 patients, all as part of an esophageal specimen and identified as malignant on routine pathological analysis.

Patients 1 and 10, both of whom had neoadjuvant chemoradiation and preoperative PET/CT findings of fludeoxyglucose avidity, had negative regional LNs on histological analysis, including a PET-avid $\mathrm{NIR}^{+}$left lower periesophageal $\mathrm{LN}$ identified at the time of surgery in patient 1 and a PET-avid left gastrohepatic LN in patient 10. A total of $3 \mathrm{NIR}^{+}$left gastric LNs were identified and removed in patient 10 , all of which were negative on routine histopathological and immunohistochemical analyses. Of note, however, despite otherwise negative preoperative PET/CT findings (aside from the avidity in the gastrohepatic region), this patient did have evidence of nodal disease in 7 local LNs removed with the esophageal specimen.

\section{DISCUSSION}

This pilot trial is a "first in human" study to develop the technique and demonstrate the feasibility of NIR image-guided lymphatic mapping as the sole modality for the identification of regional LNs during minimally invasive esophagectomy (Video 1). Although high background at the site of peritumoral injection prevented identification of local LNs removed with the esophageal specimen itself, discrete regional LNs distinct from the esophageal specimen were identified in 6 of the 9 patients who underwent esophagectomy, and in all patients in whom ICG:HSA was used as the lymphatic tracer. As seen with lung NIR imaging, peritumoral injection of ICG:HSA improved $\mathrm{NIR}^{+} \mathrm{LN}$ identification, with $\mathrm{NIR}^{+}$nodes detected in all
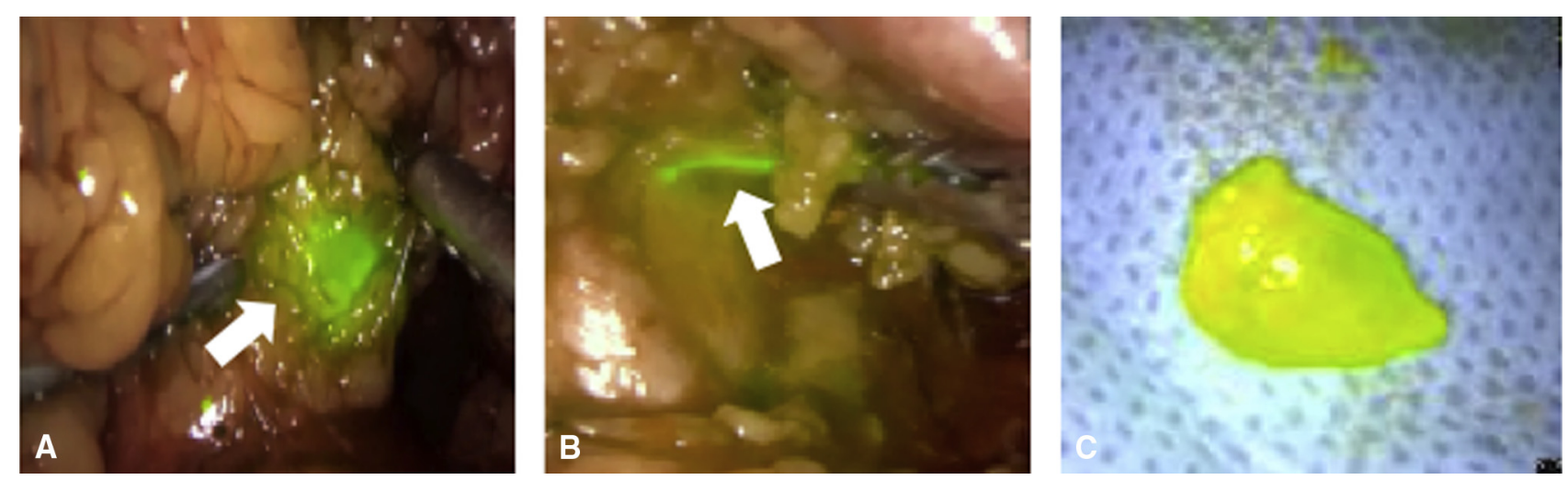

FIGURE 3. In vivo and ex vivo NIR image-guided identification of intra-abdominal esophageal LNs. A, In vivo NIR ${ }^{+}$left periesophageal LN. B, NIR ${ }^{+}$ lymphatic tract to LN. C, Ex vivo confirmation of an $\mathrm{NIR}^{+} \mathrm{LN}$. 


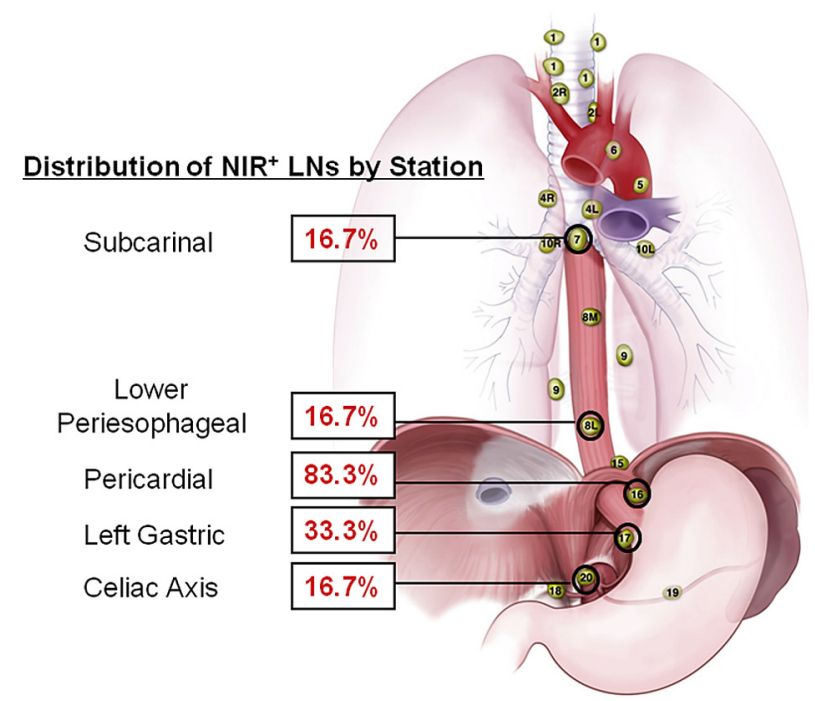

FIGURE 4. Distribution of $\mathrm{NIR}^{+} \mathrm{LNs}$ in the study patients displayed by anatomic nodal station. $N I R^{+}$, Detection of near-infrared fluorescence; $L N$, lymph node.

4 esophagectomies, whereas injection of ICG alone led to $\mathrm{NIR}^{+} \mathrm{LN}$ identification in only 2 of 5 cases.

Among the 6 patients in whom $\mathrm{NIR}^{+}$LNs were identified, 5 had abdominal $\mathrm{NIR}^{+}$LNs, whereas only 2 had thoracic $\mathrm{NIR}^{+}$LNs. The distribution of $\mathrm{NIR}^{+}$LNs in our patients is relatively consistent with the literature describing the metastatic patterns of LN metastases in lower esophageal and GE junction cancers, with an $\mathrm{NIR}^{+}$ pericardial node draining the tumor in $>80 \%$ of the patients studied. ${ }^{19-21}$ In 181 cases of lower thoracic and GE junction adenocarcinomas treated with surgery alone or neoadjuvant chemoradiation and surgery, Castoro and colleagues ${ }^{19}$

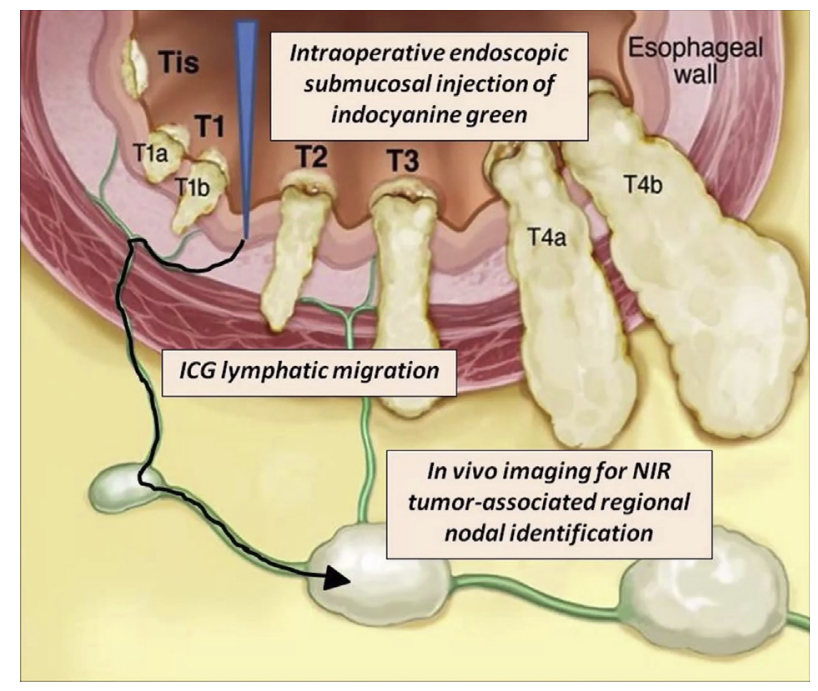

VIDEO 1. Intraoperative near-infrared image-guided technique for regional node identification in esophageal cancer. Video available at http://www.jtcvsonline.org/article/S0022-5223(16)30083-6/addons.
TABLE 3. Location of histologically detected metastatic LNs in patients with identified $\mathrm{NIR}^{+}$LNs

\begin{tabular}{lcc}
\hline & \multicolumn{2}{c}{ No. of metastatic/total nodes identified } \\
\cline { 2 - 3 } Patient & Regional $^{*}$ & Local \\
\hline 7 & $0 / 5$ & $1 / 28$ \\
8 & $0 / 8$ & $1 / 17$ \\
9 & $0 / 10$ & $1 / 15$ \\
10 & $0 / 6$ & $7 / 25$ \\
\hline
\end{tabular}

*Includes $\mathrm{NIR}^{+}$regional LNs.

identified lymphatic metastasis predominantly in regional abdominal and thoracic periesophageal nodes, including pericardial $(12.5 \%-37.1 \%)$, perigastric $(18.8 \%-35.6 \%)$, celiac axis $(14.4 \%-18.8 \%)$, thoracic periesophageal (21.9\%-29.6\%), and subcarinal (5.3\%-9.4\%) stations. The similar distribution of regional nodes identified using NIR imaging suggests that this technology may reflect the relevant tumor-draining lymphatic pathways in this disease, rather than the preferential identification of $\mathrm{NIR}^{+}$nodes in the first cavity examined. The ability to identify and characterize these pathways has the potential to improve the likelihood of an oncologic LAD by identifying the regional LNs most likely to contain metastatic disease.

At present, the inability to accurately identify and sample the appropriate LNs in a systematic fashion for esophageal cancer limits clinical decision making. NIR identification of these regional nodes ensures removal and, with further validation of their relationship to the primary tumor, may allow greater pathological and molecular analysis of the lymphatic pathway(s) leading away from the tumor, and ultimately permit better staging and identification of patients who may benefit from neoadjuvant therapy before esophagectomy is performed. Although peritumoral NIR background likely obscured the ability to detect $\mathrm{NIR}^{+}$ nodes on the esophagus directly adjacent to the tumor, van de Ven and associates ${ }^{20}$ reported that $\sim 30 \%$ of GE junction tumors also have LN metastasis at other sites beyond the peritumoral region, and Hosch and colleagues ${ }^{22}$ reported a $43 \%$ rate of "skip" metastases (defined as nodes $3 \mathrm{~cm}$ beyond the primary lesion). Failure to remove these metastatic regional nodes with the esophagectomy specimen results in a falsely low $\mathrm{N}$ stage in the current staging system, given that nodal staging is based on the number of metastatic LNs identified, rather than on the nodal location or distance that the metastases have traveled within the lymphatic system. Therefore, these studies suggest that NIR lymphatic mapping of regional LNs could be very useful in identifying regional metastatic nodes that are not removed with the specimen and result in understaging and undertreating of metastatic nodal disease. With initial safety and feasibility now established, NIR lymphatic mapping can permit targeted LN sampling of these LNs that might otherwise be missed. With accurate NIR localization of regional tumor-associated LNs and 
histological classification of local and regional LN tiers, we envision that the LN station relative to the primary tumor will take on a greater role in prognosis, as has been seen with many other solid organ tumors.

In the present study, $\mathrm{NIR}^{+}$regional LNs were negative for metastatic disease, consistent with the status of all other regional LNs removed from these patients and reflective of early stage in these surgically resectable patients. Our findings suggest that histopathological examination of $\mathrm{NIR}^{+}$LNs separate from the esophageal specimen may help guide adequate LN sampling for staging. It is of interest that several PET-avid regional nodes identified as high risk for metastases on the preoperative PET imaging also resided within the lymphatic basins mapped by peritumoral ICG and identified as $\mathrm{NIR}^{+}$. This finding suggests that these reactive nodes are draining the tumor basin and thus indeed are at high risk for occult metastases; however, none of the $\mathrm{NIR}^{+} \mathrm{LNs}$, including those retrieved from PET-avid nodal regions, stained positive for micrometastatic disease on immunohistochemical analysis. Given that PET avidity is not visible intraoperatively, the ability to use NIR imaging as an intraoperative guide to these high-risk nodes may prove very helpful if this correlation persists in future studies.

Although the prognostic value of micrometastatic disease in esophageal cancer is not firmly established, there is growing evidence that nodal micrometastasis may confer poor prognosis and even provide additional prognostic information over node-positive cases identified by hematoxylin and eosin. ${ }^{23,24}$ Interestingly, none of our patients found to have metastatic disease in local paraesophageal nodes demonstrated fludeoxyglucose avidity adjacent to the esophagus on preoperative PET/CT, even where disease was ultimately found. This finding highlights the limitations of PET/CT for reliably identifying metastatic LNs and suggests that additional imaging tools, such as dose-adjusted NIR lymphatic mapping, may improve the identification of LNs for increased scrutiny to rule out metastasis that cannot be detected by other current imaging modalities.

The present study is the first human trial to develop a technique for in situ minimally invasive NIR-guided lymphatic mapping in esophageal cancer and to directly compare ICG alone and ICG:HSA. Previous SLN mapping trials comparing ICG and ICG:HSA in breast and cervical cancers suggested no significant difference in the $\mathrm{NIR}^{+}$ LN yield ${ }^{25,26}$; however, ICG mixed with HSA has been shown to increase the hydrodynamic diameter of the dye, improving fluorescence and nodal retention in a porcine model of SLN mapping. ${ }^{18}$ Previous studies by our laboratory and others have shown that HSA is variably required for NIR imaging based on the tissue to be imaged. ${ }^{13,14,27}$ The sole published study on esophageal NIR lymphatic mapping using ICG required the addition of preoperative CT lymphography with iopamidol to guide nodal identification, but the $\mathrm{NIR}^{+}$nodal identification rate was $95 \%$ when assessed via open thoracotomy. ${ }^{17}$ The periesophageal background was not discussed, however. Thus, a randomized comparison of ICG and ICG:HSA was included in the current trial design to identify the more promising tracer for esophageal lymphatic mapping.

$\mathrm{NIR}^{+}$LNs were not identified in 3 of the 9 patients who underwent esophagectomy, and, surprisingly, ICG suspended in sterile water without albumin was used in all 3 of these patients, suggesting that albumin enhances ICG retention in human LNs as well, at least in the thoracic cavity. In our 3 ICG alone patients in whom $\mathrm{NIR}^{+}$nodes were not identified, abdominal imaging occurred between 30 minutes and 1 hour after dye injection and lasted for 2 to 2.5 hours, consistent with other cases in the trial that did lead to NIR nodal identification. It is possible that rapid lymphatic clearance and poor lymphatic retention with ICG alone led to our missing the relevant LNs during the later thoracic component, which typically began about 4 hours after injection. We hypothesize that ICG:HSA may be preferable in esophageal cancer lymphatic mapping, because this formulation may remain within the lymphatics and regional $\mathrm{LNs}$ for the longer periods required for esophagectomy and LAD.

NIR background on the esophageal specimen, as a result of transmucosal dye migration and dye spillage during dissection, might have precluded the intraoperative identification of local $\mathrm{NIR}^{+}$LNs on the specimen. Because the ICG dose used in this trial $(2.5 \mathrm{mg})$ was initially optimized for lung cancer lymphatic mapping, where background levels are lower, we hypothesize that ICG dose reduction will significantly decrease the peritumoral background and enhance the in vivo signal-to-noise ratio, thereby improving identification of $\mathrm{NIR}^{+}$local LNs. The ability to accurately sample tumor-associated nodes for histological scrutiny before esophagectomy, thus avoiding the need for complete esophagectomy and LAD with back table nodal dissection, as has been required in studies using radiocolloid approaches, would permit in situ staging and possible selection of truly N0 patients for esophagealsparing endomucosal resection. ${ }^{10,12}$ Given that a significant number of early lesions may have nodal metastasis, which will be missed by PET imaging, an optimized NIR-guided 2-field LN mapping technique may be justified as a means to accurately assess the pathological status of both local and regional $\mathrm{NIR}^{+} \mathrm{LNs}$ in situ.

Given the theoretical risk of lymphatic ablation, and in particular considering reports of poor SLN identification after neoadjuvant treatment with other techniques, we were concerned that NIR imaging for lymphatic mapping in esophageal cancer might be limited by previous neoadjuvant radiation treatment. ${ }^{18}$ Interestingly, this was not the 
case; $\mathrm{NIR}^{+}$nodes were readily identified in the 3 neoadjuvant patients given ICG:HSA, providing further support for the use of ICG:HSA as the NIR fluorophore in patients with esophageal cancer. We recognize that this pilot trial includes only a small patient sample, and that future trials are needed to truly determine the impact of neoadjuvant treatment on $\mathrm{NIR}^{+} \mathrm{LN}$ yield and the ability to identify the correct tumor-associated nodes.

Limitations of the present trial include its small sample size without identification of a large number of diseasepositive regional LNs to assess the accuracy of NIR regional nodal mapping. In addition, cervical and upper mediastinal LNs were not assessed in most cases, because 3-field LAD was not performed given the potential risks of further dissection in patients without clinical or radiographic evidence of disease. Although not sufficiently powered to definitively compare the performance of ICG:HSA to that of ICG alone, this pilot trial can aid a preliminary determination of which tracer has the best likelihood of success, such that a larger trial can focus on identifying the optimal dose of ICG:HSA to minimize the peritumoral background on the esophageal specimen.

Future efforts in NIR lymphatic mapping in esophageal cancer will focus on improving the identification of both regional and adjacent local esophageal LNs, such that in situ nodal staging may ultimately permit organ preservation for patients deemed node-negative. The development of innovative dual-modality approaches pairing NIR lymphatic mapping with other novel tracers may more directly identify suspicious nodes and enhance the detection of nodal metastases. Promising approaches, such as preoperative magnetic resonance imaging with intravenous superparamagnetic iron oxide particles, have demonstrated feasibility for LN staging in small pilot trials in other malignancies and merit further evaluation in intrathoracic malignancies, such as esophageal cancer, and the present trial has established the groundwork for these upcoming studies. ${ }^{28}$ Further optimization of minimally invasive NIR lymphatic mapping for esophageal cancer has the potential to provide a safe and easy to use intraoperative approach to the identification of tumor-specific regional LNs for improved targeted staging.

\section{Conflict of Interest Statement}

Dr Wee reports consulting fees from Medtronic. All other authors have nothing to disclose with regard to commercial support.

The authors thank Marcia Williams for preparing the illustrations in Figures 2 and 4.

\section{References}

1. National Cancer Institute. Surveillance, Epidemiology, and End Results Program. Available at: http://seer.cancer.gov/statfacts/html/esoph.html. Accessed April 19, 2016.
2. Talsma K, van Hagen P, Grotenhuis BA, Steyerberg EW, Tilanus HW, van Lanschot JJ, et al. Comparison of the 6th and 7th editions of the UICC-AJCC TNM classification for esophageal cancer. Ann Surg Oncol. 2012;19:2142-8

3. Altorki NK, Zhou XK, Stiles B, Port JL, Paul S, Lee PC, et al. Total number of resected lymph nodes predicts survival in esophageal cancer. Ann Surg. 2008; 248:221-6.

4. Edge S, Byrd DR, Compton CC, Fritz AG, Greene FL, Trotti A. Esophagus and esophagogastric junction. In: AJCC Cancer Staging Manual. 7th ed. New York: Springer; 2010:103-16.

5. Herrera LJ. Extent of lymphadenectomy in esophageal cancer: how many lymph nodes is enough? Ann Surg Oncol. 2010;17:676-8.

6. Greenstein AJ, Litle VR, Swanson SJ, Divino CM, Packer S, Wisnivesky JP. Effect of the number of lymph nodes sampled on postoperative survival of lymph node-negative esophageal cancer. Cancer. 2008;112:1239-46.

7. Peyre CG, Hagen JA, DeMeester SR, Altorki NK, Ancona E, Griffin SM, et al. The number of lymph nodes removed predicts survival in esophageal cancer: an international study on the impact of extent of surgical resection. Ann Surg. 2008; 248:549-56.

8. Lerut T, Nafteux P, Moons J, Coosemans W, Decker G, De Leyn P, et al. Three-field lymphadenectomy for carcinoma of the esophagus and gastroesophageal junction in $174 \mathrm{R} 0$ resections: impact on staging, disease-free survival, and outcome: a plea for adaptation of TNM classification in upper-half esophageal carcinoma. Ann Surg. 2004;240:962-72.

9. Nishihira T, Hirayama K, Mori S. A prospective randomized trial of extended cervical and superior mediastinal lymphadenectomy for carcinoma of the thoracic esophagus. Am J Surg. 1998;175:47-51.

10. Takeuchi H, Kawakubo H, Takeda F, Omori T, Kitagawa Y. Sentinel node navigation surgery in early-stage esophageal cancer. Ann Thorac Cardiovasc Surg. 2012;18:306-13.

11. Uenosono Y, Arigami T, Yanagita S, Kozono T, Arima H, Hirata M, et al. Sentinel node navigation surgery is acceptable for clinical T1 and N0 esophageal cancer. Ann Surg Oncol. 2011;18:2003-9.

12. Thompson SK, Bartholomeusz D, Jamieson GG. Sentinel lymph node biopsy in esophageal cancer: should it be standard of care? J Gastrointest Surg. 2011;15: 1762-8.

13. Gilmore DM, Khullar OV, Jaklitsch MT, Chirieac LR, Frangioni JV, Colson YL. Identification of metastatic nodal disease in a phase I dose-escalation trial of intraoperative sentinel lymph node mapping in non-small cell lung cancer using near-infrared imaging. J Thorac Cardiovasc Surg. 2013;146:562-70.

14. Gilmore DM, Khullar OV, Gioux S, Stockdale A, Frangioni JV, Colson YL, et al Effective low-dose escalation of indocyanine green for near-infrared fluorescent sentinel lymph node mapping in melanoma. Ann Surg Oncol. 2013;20:2357-63.

15. Mitsumori N, Nimura H, Takahashi N, Kawamura M, Aoki H, Shida A, et al. Sentinel lymph node navigation surgery for early stage gastric cancer. World $J$ Gastroenterol. 2014;20:5685-93.

16. Troyan SL, Kianzad V, Gibbs-Strauss SL, Gioux S, Matsui A, Oketokoun R, et al. The FLARE intraoperative near-infrared fluorescence imaging system: a first-in-human clinical trial in breast cancer sentinel lymph node mapping. Ann Surg Oncol. 2009;16:2943-52.

17. Yuasa Y, Seike J, Yoshida T, Takechi H, Yamai H, Yamamoto Y, et al. Sentinel lymph node biopsy using intraoperative indocyanine green fluorescence imaging navigated with preoperative CT lymphography for superficial esophageal cancer. Ann Surg Oncol. 2012;19:486-93.

18. Ohnishi S, Lomnes SJ, Laurence RG, Gogbashian A, Mariani G, Frangioni JV. Organic alternatives to quantum dots for intraoperative near-infrared fluorescent sentinel lymph node mapping. Mol Imaging. 2005;4:172-81.

19. Castoro C, Scarpa M, Cagol M, Ruol A, Cavallin F, Alfieri R, et al. Nodal metastasis from locally advanced esophageal cancer: how neoadjuvant therapy modifies their frequency and distribution. Ann Surg Oncol. 2011;18: 3743-54.

20. van de Ven C, De Leyn P, Coosemans W, Van Raemdonck D, Lerut T. Three-field lymphadenectomy and pattern of lymph node spread in T3 adenocarcinoma of the distal esophagus and the gastro-esophageal junction. Eur J Cardiothorac Surg. 1999;15:769-73.

21. Stein HJ, Feith M, Bruecher BL, Naehrig J, Sarbia M, Siewert JR. Early esophageal cancer: pattern of lymphatic spread and prognostic factors for long-term survival after surgical resection. Ann Surg. 2005;242:566-73.

22. Hosch SB, Stoecklein NH, Pichlmeier U, Rehders A, Scheunemann P, Niendorf A, et al. Esophageal cancer: the mode of lymphatic tumor cell spread and its prognostic significance. J Clin Oncol. 2001;19:1970-5. 
23. Prenzel KL, Hölscher AH, Drebber U, Agavonova M, Gutschow CA, Bollschweiler E. Prognostic impact of nodal micrometastasis in early esophageal cancer. Eur J Surg Oncol. 2012;38:314-8.

24. Zingg U, Montani M, Busch M, Metzger U, Went P, Oertli D. Prognostic influence of immunohistochemically detected lymph node micrometastasis and histological subtype in pNO oesophageal cancer. Eur J Surg Oncol. 2009;35: 593-9.

25. Polom K, Murawa D, Nowaczyk P, Rho YS, Murawa P. Breast cancer sentinel lymph node mapping using near infrared guided indocyanine green and indocyanine green-human serum albumin in comparison with gamma emitting radioactive colloid tracer. Eur J Surg Oncol. 2012;38:137-42.

26. Schaafsma BE, van der Vorst JR, Gaarenstroom KN, Peters AA, Verbeek FP, de Kroon CD, et al. Randomized comparison of near-infrared fluorescence lymphatic tracers for sentinel lymph node mapping of cervical cancer. Gynecol Oncol. 2012;127:126-30.

27. Yamashita S, Tokuishi K, Miyawaki M, Anami K, Moroga T, Takeno S, et al. Sentinel node navigation surgery by thoracoscopic fluorescence imaging system and molecular examination in non-small cell lung cancer. Ann Surg Oncol. 2012;19:728-33.

28. McDermott S, Thayer SP, Fernandez-Del Castillo C, Mino-Kenudson M, Weissleder R, Harisinghani MG. Accurate prediction of nodal status in preoperative patients with pancreatic ductal adenocarcinoma using next-gen nanoparticle. Transl Oncol. 2013;6:670-5.

Key Words: esophageal cancer, near-infrared imaging, sentinel lymph node

Readers who found these articles interesting may also like to read the following papers found in recent and future issues of our sister publications, Seminars in Thoracic and Cardiovascular Surgery and Operative Techniques in Thoracic and Cardiovascular Surgery!

\section{Thoracic: Other Thoracic Surgical Papers}

News and Views: Recent and Future Developments in Chest Wall Reconstruction. Calvin Ng. Semin Cardiovasc Surg 2015; Summer; 27(2):234-239.

Current Readings: What the Surgeon Needs to Know About Databases. Alessandro Brunelli. Semin Thorac Cardiovasc Surg 2015; Summer; 27(2):251-256.

Original Submission: Endobronchial Treatment of bronchopleural fistulas by using Intrabronchial valve system: a case series. Eitan Podgaetz. Semin Thorac Cardiovasc Surg 2015; Summer; 27(2):218-222.

Editorial Commentary: One-Way Valves to the Rescue or Bronchopleural Fistulae. Eric J. Seeley. Semin Thorac Cardovasc Surg 2015; Summer; 27(2):223-224.

Tracheal Surgery: Posterior Splinting Tracheoplasty for Tracheomalacia. Cameron D. Wright. Oper Tech Thorac Cardiovasc Surg 2015; Spring; 20(1):31-45.

Laryngeal Resection and Reconstruction. John D. Mitchell. Oper Tech Thorac Cardiovasc Surg 2015; Spring; 20(1):46-62.

Video-Assisted Thoracoscopic Segmentectomy of the Lower Lobe: Superior and Basilar Segmentectomy. Nathaly P. Llore. Oper Tech Thorac Cardiovasc Surg 2015; Summer; 20(2):162-175.

Robotic First Rib Resection. Eric Strother. Oper Tech Thorac Cardiovasc Surg 2015; Summer; 20(2):178-188.

Surgical management of esophageal perforation. Manu Sancheti. Oper Tech Thorac Cardiovasc Surg 2015; Autumn; 20(3):234-250.

Endoscopic techniques for the management of esophageal perforation. Shanda Blackmon. Oper Tech Thorac Cardiovasc Surg 2015; Autumn; 20(3):251-278. 